\title{
Computer Modelling of a Vehicle System
}

\author{
M. Prem Jeya Kumar'*, J. Sandeep Anand ${ }^{2}$, K. Gopalakrishnan ${ }^{3}$, B. Satheesh ${ }^{4}$ and R. Anbazhagan ${ }^{5}$ \\ 'Professor, Department of Automobile Engineering, Bharath University, Chennai-73; \\ premjeyakumar.coe@bharathuniv.ac.in \\ ${ }^{2}$ Professor, Department of Computer Science Engineering, Bharath University, Chennai-73; \\ sundeepssn@hotmail.com \\ ${ }^{3}$ Professor of Electronics Communications Engineering, Bharath University, Chennai-73; \\ gopikrishna2804@gmail.com \\ 4Professor of Electronics Communications Engineering, Bharath University, Chennai-73; \\ satheesh.mit@gmail.com \\ ${ }^{5}$ Professor, Department of Automobile Engineering, Bharath University, Chennai-73; \\ anbu.mit@gmail.com
}

\begin{abstract}
The purpose of computer modeling of a vehicle system is to develop basic methods for computer formulation and solution of the equations of motion. This requires systematic techniques for formulating the equations and numerical methods for solving them. The computer program developed here for the analysis of vehicle system dynamics is a special purpose program. It deals with only a specific type of application say lateral stability or dynamic response. The equations of motion for that particular application are derived a priori and then formulated into the program. As input to the program, the various data's like, mass of the entire vehicle, sprung mass, unstrung mass etc., the initial values of parameters of a vehicle system have been provided. Such a special purpose program can be made computationally efficient and its storage requirement can be minimized with the result that it will be suitable for implementation on small personal computers.
\end{abstract}

Keywords: Dynamics, Linear Modeling, Sprung Mass, Unstrung Mass, Subroutine Mod, Stiffness

\section{Introduction}

Computer modeling is concerned with the solution of the equations of motion that were formulated for the different movements of the vehicles. This formulation permits the application of many mathematical and numerical techniques. It may be noted that the stability analysis is restricted to linear models. Hence if a motion is stable for a given set of initial conditions and input, it cannot be implied that the motion will remain stable for other sets of initial conditions and inputs. Hence great care is required in the stability analysis and in this connection it is useful to employ appropriate stability theory [1-15].

* Corresponding author:

M. Prem Jeya Kumar (premjeyakumar.coe@bharathuniv.ac.in)
There exist several concepts of stability such as stability in the sense of Lagrange, Poincare, Yaupon, bounded ness of response, and input - output stability. For a particular application, one concept may be unduly restrictive, whereas another may have no physical significance. The choice of one of these concepts of stability depends on its physical significance in a particular application. Realizing the complexities involved, it s thought to restrict the study of stability analysis to linear systems. It is well known that for the solution of linear time invariant equations of motion, one can employ either the normal mode superposition method or the direct numerical integration methods [16-27]. 


\section{Linear Modelling}

The linear computer modeling is developed mainly to study the lateral stability, analysis of the vehicle system. Here the equations of motion corresponding to a vehicle system are taken into consideration.

In the linear model, the equations of motion can basically be condensed into the following form.

$$
[\mathrm{M}]\{\ddot{\mathrm{x}}\}+[\mathrm{C}]\{\dot{\mathrm{x}}\}+[\mathrm{k}]\{\mathrm{x}\}=\{0\}
$$

Where, $[M],[C]$ and $[K]$ are mass, damping and stiffness matrices respectively. $\{\mathrm{x}\},\{\dot{\mathrm{x}}\}$ and $\{\ddot{\mathrm{X}}\}$ are displacement, velocity and acceleration vector of motion. For linear model, since the properties of the element do not change with time, the mass, damping and stiffness matrices will be constant and time invariant.

\section{Stability Analysis Program (SAP)}

The $\mathrm{C}$ program for stability analysis is developed. The program is developed in modular form in $\mathrm{C}$ language. It should run on most of the $\mathrm{C}$ compilers without any difficulty. However, some compilers may require minor modifications in the program [28-42].

The main routine of the program performs three major tasks.

(a) Reading the input data, either directly or calling other input subroutines

(b) Generating inertia, damping and stiffness matrices $[\mathrm{M}],[\mathrm{C}]$ and $[\mathrm{K}]$ using input data and then finding the dynamic matrix by calling subroutines

(c) Carrying out stability analysis by calling concerned subroutines and writes the result in DAT file format.

\subsection{Subroutine INIT}

This subroutine gives the information describing the initial values of various parameters of a vehicle system which are required for stability analysis.

\subsection{Subroutine MOD}

This subroutine is corresponding to the model. It is used to generate and assemble mass, damping and stiffness matrices characterizing the vehicle system. This subroutine will vary for different systems and will depend on the equations of motion.

\subsection{Subroutine VALUES}

This subroutine is used to display the values of parameters on the monitor or printer or both in a particular format. Hence it is easy to see the values of various parameters together in a particular form.

\subsection{Subroutine CHANGE}

This subroutine is used to change the initial values of parameters whenever necessary. Then the values are changed this subroutine will display the new values of parameters on the monitor or printer or both.

\subsection{Subroutine MATMUL}

This subroutine is used to multiply two matrices and store the elements of the final matrix Here $\mathrm{D}(\mathrm{M}, \mathrm{K})=\mathrm{A}(\mathrm{M}, \mathrm{N})$ ${ }^{*} \mathrm{~B}(\mathrm{~N}, \mathrm{~K})$.

\subsection{Subroutine MATDYN}

This subroutine is used to assemble the dynamic matrix from the inertia, damping and stiffness matrices as per the relations given earlier.

\subsection{Function INVER}

This function routine is used to find the inverse of the inertia matrix. Since the inertia matrix is a diagonal matrix, the inverse of the matrix is also having only diagonal elements. In inverse matrix, each element in the diagonal will be equal to the reciprocal of the corresponding element of the original matrix.

\subsection{Subroutine RNAA}

This subroutine computes all the Eigen values and their corresponding Eigenvectors of an arbitrary real matrix. The matrix is balanced by exact norm reducing similarity transformations and then is reduced to Heisenberg form by elementary similarity transformation. The program which is used for the stability analysis is an interactive type. First it will identify what type of analysis is to be carried out and get the data from the input file. Then it will find values of all the elements of matrices $[\mathrm{M}],[\mathrm{C}]$ 
and $[\mathrm{K}]$, and using these matrices it will do the analysis and gives the Eigen values for the particular set of inputs. The computer modeling provides the methodology to carryout the lateral stability analysis of an automotive vehicle.

\section{Tyre Modeling Flowchart}

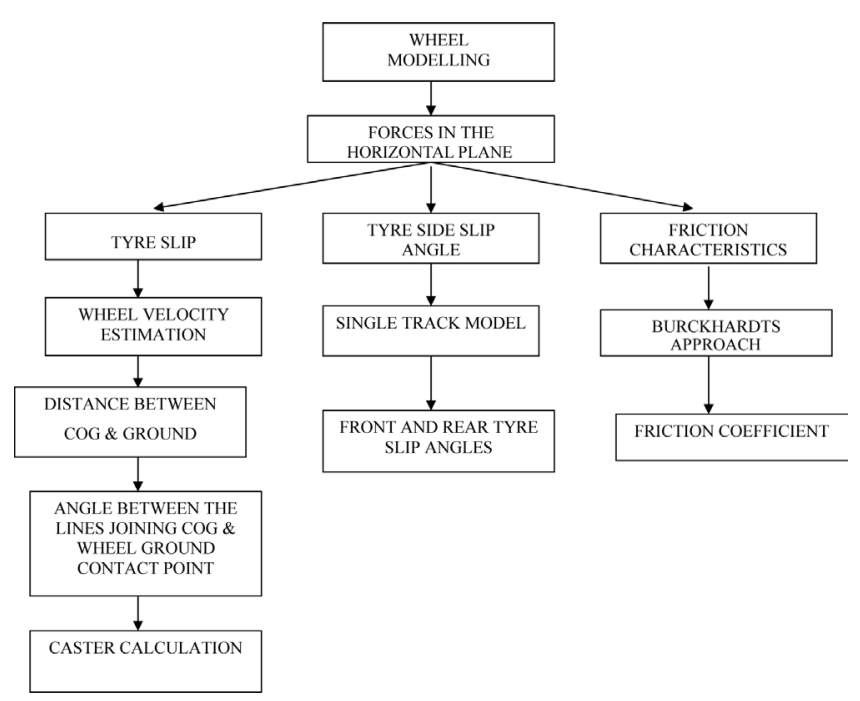

Figure 1.1 Flowchart of the procedure and different parameters involved in tyre modeling.

\section{Slip Calculation}

Wheel slip occurs due to the difference of the rotational equivalent of tyre velocity $(\boldsymbol{V} \boldsymbol{r})$ and the translator's velocity $(V w)$ of the wheel. The acceleration and braking forces are produced due to the presence of slip. There are two types of slip,

1. Longitudinal slip

2. Lateral slip

If the tyre rotates without lateral slip, tyre slip is the difference between the rotational equivalent velocity and translator velocity. But the longitudinal slip and lateral slip occur simultaneously. Usually Longitudinal slip is considered in the direction of motion of the wheel (i.e.) in the direction of wheel velocity and the lateral slip in the direction perpendicular to the direction of wheel velocity. The rotational equivalent wheel velocity is in the direction of $\mathrm{X}$ axis of the wheel coordinate system whereas the wheel velocity is inclined at tyre side slip angle to it. It is evident from the following figure. Hence $V_{r}$ should be multiplied by the cosine of tyre side slip angle before calculating slip. In slip calculations the speed difference is divided the respective larger speed to avoid division by zero.

The slip is calculated for driving and braking conditions for all the four wheels.

\section{Braking}

$$
\begin{aligned}
& \text { Longitudinal slip } s_{l i j}=\frac{v_{r i j} \cos \alpha_{i j}-v_{w i j}}{v_{w i j}} \\
& \text { Lateral slip } \quad s_{s i j}=\frac{v_{r i j} \sin \alpha_{i j}}{v_{w i j}}
\end{aligned}
$$

\section{Driving}

$$
\begin{aligned}
& \text { Longitudinal slips } s_{l i j}=\frac{v_{r i j} \cos \alpha_{i j}-v_{w i j}}{v_{r i j} \cos \alpha_{i j}} \\
& \text { Lateralslip } \quad s_{s i j}=\tan \alpha_{i j}
\end{aligned}
$$

Resultant slip is given by

$$
s_{\text {resij }}=\sqrt{s_{l i j}^{2}+s_{s i j}^{2}}
$$

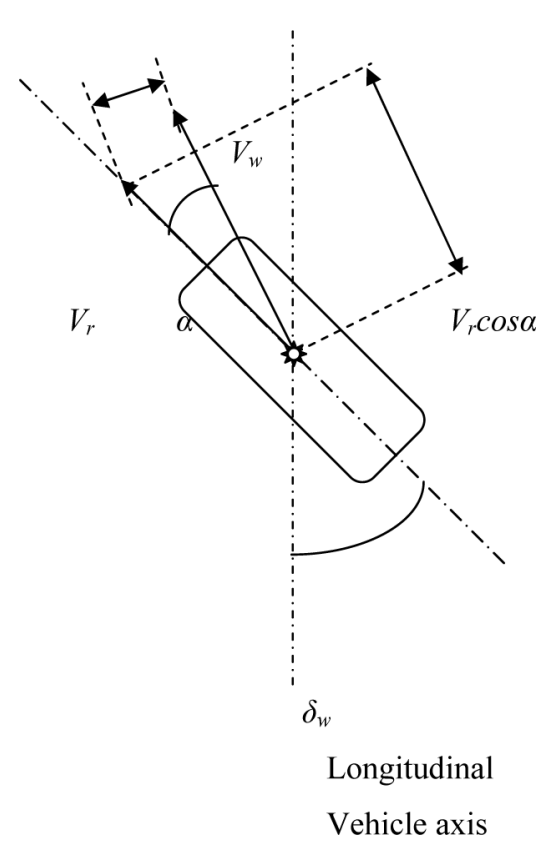

Figure 1.2 Wheel slip calculation. 
$\mathbf{s}_{\text {resij }}$ Resultant slip

$\mathbf{s}_{\text {lij }}$ Longitudinal slip in the direction of $v_{\text {wij }}$

$\mathbf{s}_{\text {sij }} \quad$ Lateral slip in the direction perpendicular to $v_{\text {wij }}$

$\boldsymbol{a}_{\mathrm{ij}} \quad$ Tyre side slip angle

$\mathbf{v}_{\text {rij }}$ Rotational equivalent wheel velocity of tyre

$\mathbf{v}_{\text {wij }}$ Translatory wheel velocity

The subscript ij represents the front/rear, left/right tyres.

The three variables Tyre side slip angle, Rotational equivalent velocity of tyre and the translator velocity of the tyre are calculated hereafter.

\section{Distances Between Vehicle Cog and Tyre Road Contact Points}

The point of action of tyre force is the tyre ground contact point. It does not lie at the centre of the tyre road contact patch but it lies towards the rear due to wheel caster. It is evident from the Figure 1.3. It shows an aerial view of the tyre contact area including the tyre road contact point. The middle point of the tyre contact area migrates outwards and creates a torque with the longitudinal force which during acceleration increases self aligning torque and during braking decreases it.

$\boldsymbol{X}_{w} \quad \mathrm{X}$ axis of wheel coordinate system

$Y_{w} \quad \mathrm{Y}$ axis of wheel coordinates system

$Z_{w} \quad Z$ axis of wheel coordinates system

$\boldsymbol{F}_{w l} \quad$ Frictional force in the direction of $\boldsymbol{X}_{w}$

$\boldsymbol{F}_{w s}$ Frictional force in the direction of $\boldsymbol{X}_{w}$

$\boldsymbol{n}_{\boldsymbol{l}} \quad$ Longitudinal caster

$\boldsymbol{n}_{\boldsymbol{s}} \quad$ Lateral caster

The caster is generally assumed to be constant and only the component in the direction of the wheel plane

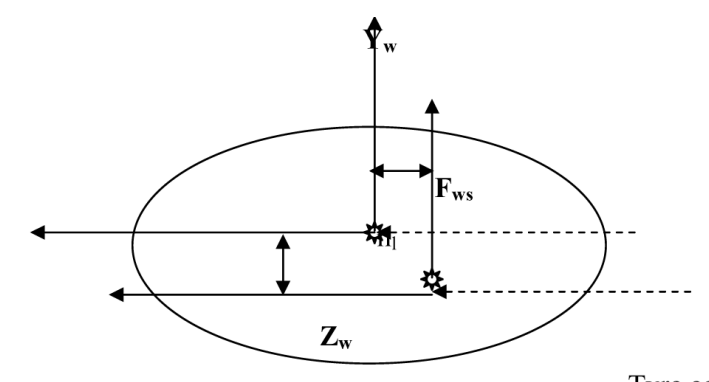

$X_{\mathrm{w}}$

$\mathrm{n}_{\mathrm{s}}$

$\mathbf{F}_{\mathrm{wl}}$

Tyre centre

Shifted tyre
Contact point

Figure 1.3 View from above of tyre contact area. is considered. But, using the above method the caster can be calculated dynamically (with direction information). The frictional forces $\boldsymbol{F}_{\boldsymbol{l}}$ and $\boldsymbol{F}_{\boldsymbol{s}}$ act in the direction of the wheel velocity $\boldsymbol{v}_{w}$ and in a direction perpendicular to it. The $\boldsymbol{F}_{w l}$ and $\boldsymbol{F}_{w s}$ can be obtained by transforming the forces $\boldsymbol{F}_{\boldsymbol{l}}$ and $\boldsymbol{F}_{s}$ into the wheel coordinate system.

The distance between the tyre ground contact point and Vehicle COG ( $\boldsymbol{r i j}$ ) can be calculated geometrically for each tyre. The angle between the line joining the Vehicle COG and the tyre/road contact point $\left(\gamma_{i j}\right)$ can also be found geometrically.

\section{Front Right Tyre}

The mathemathical model of the Front Right Tyre is as shown in the Figure 1.4

$$
\begin{aligned}
& r_{f r}=\sqrt{\left(l_{f}-n_{l f r} \cos \delta_{w}+n_{s f r} \sin \delta_{w}\right)^{2}+\left(\frac{b_{f}}{2}+n_{s f r} \cos \delta_{w}+n_{l f r} \sin \delta_{w}\right)^{2}} \\
& \gamma_{f l}=\tan ^{-1}\left(\frac{l_{f}-n_{l f r} \cos \delta_{w}+n_{s f r} \sin \delta_{w}}{\frac{b_{f}}{2}+n_{s f r} \cos \delta_{w}+n_{l f r} \sin \delta_{w}}\right)
\end{aligned}
$$

\section{Front Left Tyre}

The mathemathical model of the Front Left Tyre is as shown in the Figure 1.5

$$
\begin{aligned}
& r_{f l}=\sqrt{\left(l_{f}-n_{l f} \cos \delta_{w}+n_{s f l} \sin \delta_{w}\right)^{2}+\left(\frac{b_{f}}{2}-n_{l f l} \sin \delta_{w}-n_{s f l} \cos \delta_{w}\right)^{2}} \\
& \gamma_{f l}=\tan ^{-1}\left(\frac{\frac{b_{f}}{2}-n_{l f l} \sin \delta_{w}-n_{s f l} \cos \delta_{w}}{l_{f}-n_{l f l} \cos \delta_{w}+n_{s f l} \sin \delta_{w}}\right)
\end{aligned}
$$

\section{Rear Right Tyre}

The mathemathical model of the Rear Right Tyre is as shown in the Figure 1.6

$$
\begin{aligned}
& r_{r}=\sqrt{\left[\left(l_{r}+n_{l r r}\right)^{2}+\left(b_{r} / 2+n_{s r r}\right)^{2}\right]} \\
& \gamma_{r r}=\tan ^{-1}\left(\frac{b_{r} / 2+n_{s r r}}{l_{r}+n_{l r r}}\right)
\end{aligned}
$$




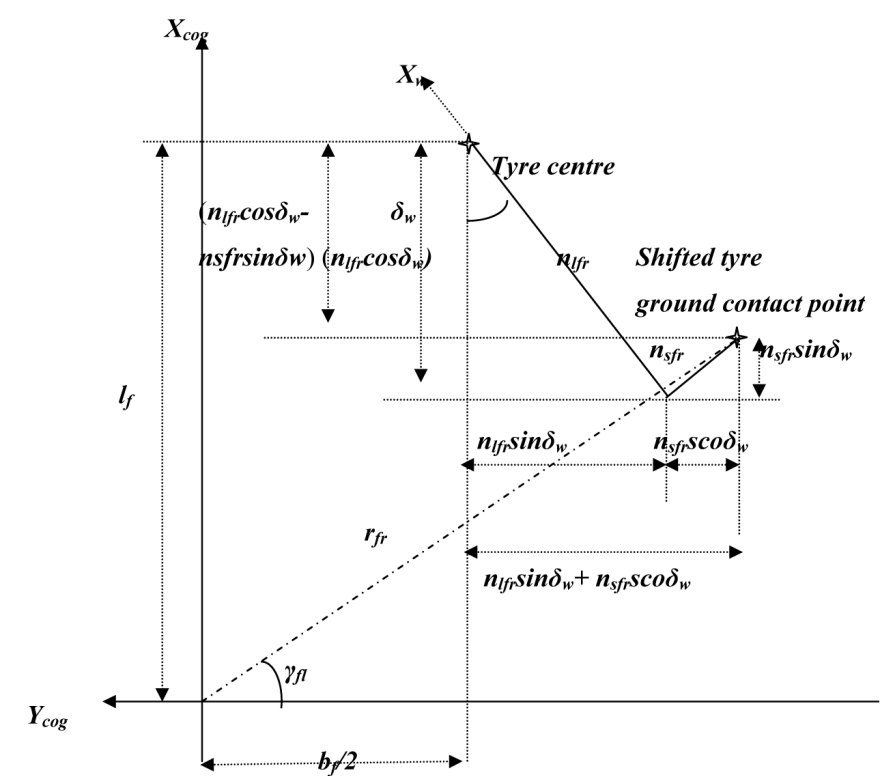

Figure 1.4 Geometric calculation of the distance $r_{f r}$

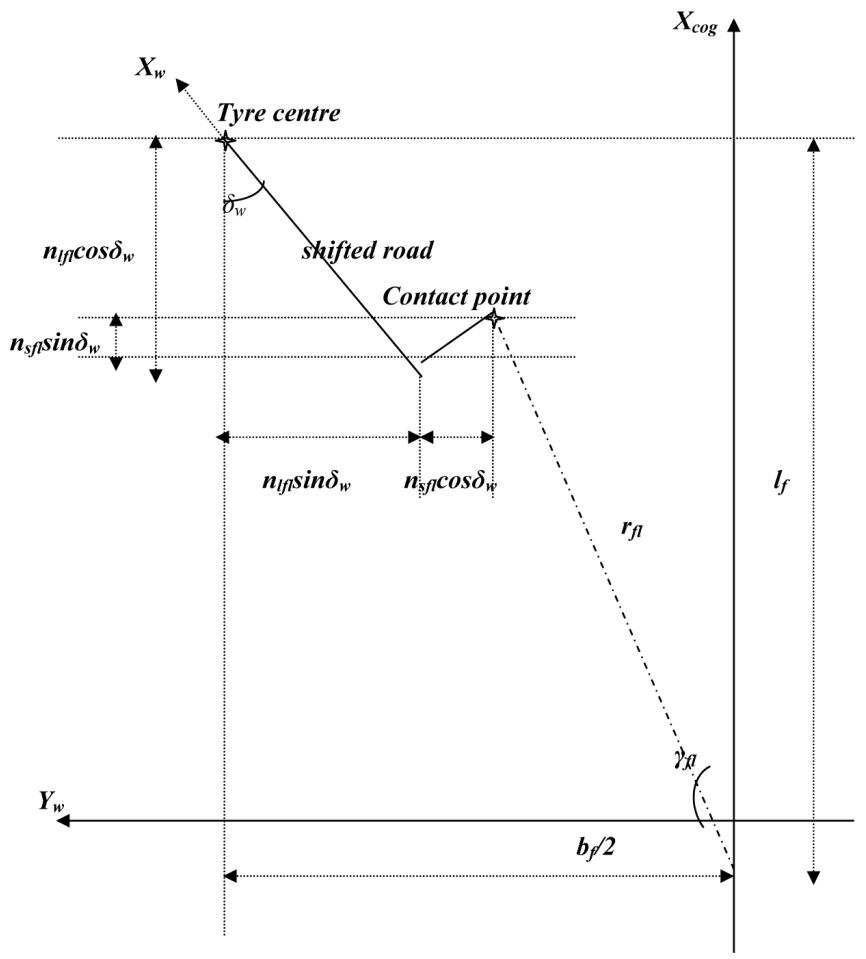

Figure 1.5 Geometric calculation of the distance rfl.

\section{Rear Left Tyre}

The mathemathical model of the Rear Left Tyre is as shown in the Figure 1.7

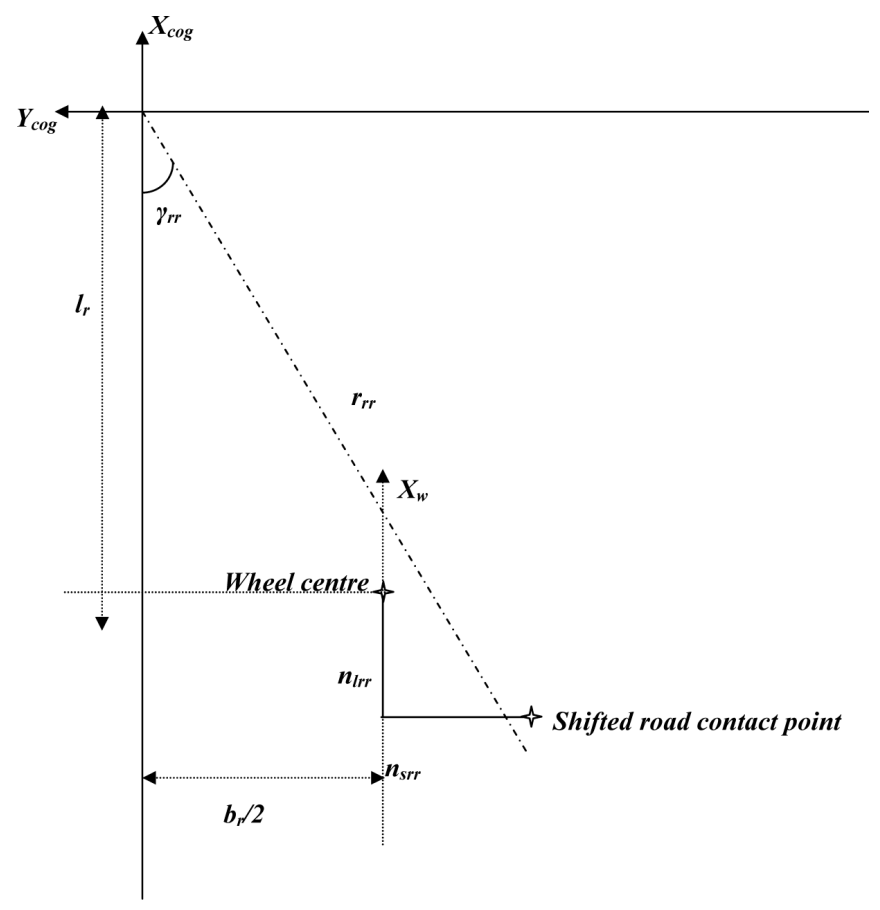

Figure 1.6 Geometric calculation of the distance $r_{r r}$.

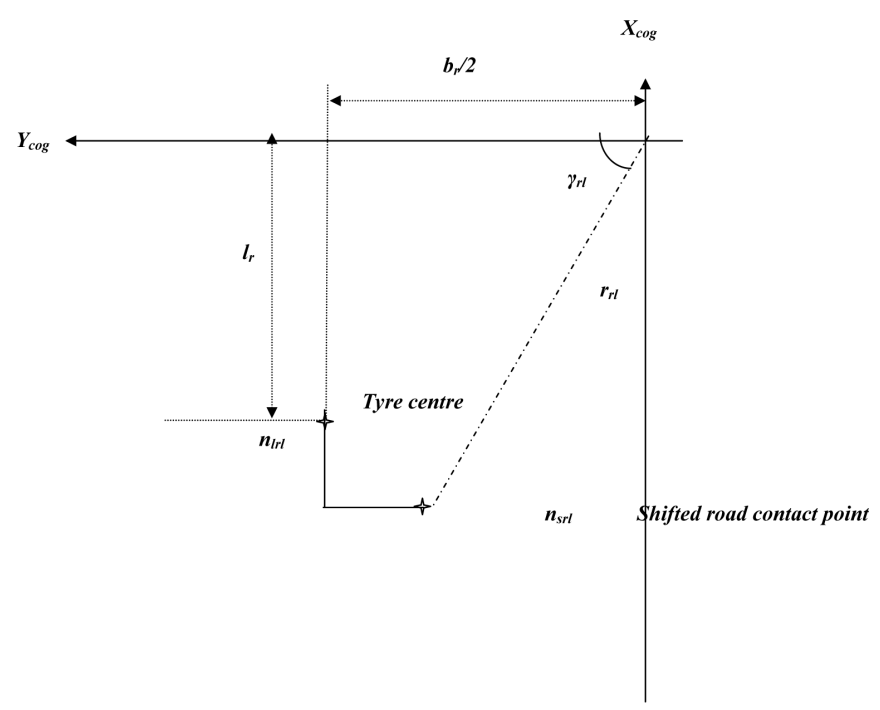

Figure 1.7 Geometric calculation of the distance $r_{r l}$

$$
\begin{aligned}
& r_{r l}=\sqrt{\left[\left(l_{r}+n_{l r l}\right)^{2}+\left(b_{r} / 2-n_{s r l}\right)^{2}\right]} \\
& \gamma_{r l}=\tan ^{-1}\left(\frac{l_{r}+n_{l r l}}{b_{r} / 2-n_{s r l}}\right)
\end{aligned}
$$

$\boldsymbol{r}_{f r}$ Distance between vehicle COG and front right tyre road contact point

$\boldsymbol{r}_{f} \quad$ Distance between vehicle COG and front left tyre road contact point 
$\boldsymbol{r}_{r l} \quad$ Distance between vehicle COG and rear left tyre road contact point

$\boldsymbol{r}_{r r}$ Distance between vehicle COG and rear right tyre road contact point

$\gamma_{f r}$ Angle between line joining vehicle COG, front right tyre road contact point and Vehicle COG coordinate system

$\gamma_{f l} \quad$ Angle between line joining vehicle COG, front left tyre road contact point and Vehicle COG coordinate system

$\gamma_{r l} \quad$ Angle between line joining vehicle COG, rear right tyre road contact point and Vehicle COG coordinate system

$\gamma_{r r}$ Angle between line joining vehicle COG, rear left tyre road contact point and Vehicle COG coordinate system

$\boldsymbol{l}_{\boldsymbol{f}}$ Distance between vehicle COG and front axle

$l_{r} \quad$ Distance between vehicle COG and rear axle

$\boldsymbol{b}_{\boldsymbol{f}}$ Distance between wheels on front axle

$\boldsymbol{b}_{r}$ Distance between wheels on rear axle

\section{Rolling Resistance Calculation}

The primary cause of rolling resistance is the hysterisis of the tyre material, due to deflection of the carcass while rolling. There are also secondary causes such as tyre/road friction, resistance due to air circulating inside the tyre. But the hysterisis loss accounts for (90-95) \% of the rolling resistance. There are a no of factors affecting tyre deformation, they are

\subsection{Tyre Construction}

Thicker treads and increased number of carcass plies result in more work to be done by the tyre while rolling thus resulting in high hysteric's losses and high rolling resistance.

\subsection{Road Surface}

Rolling resistance is less for hard, smooth and dry road surfaces. Rolling resistance is high for worn out roads since more work is done by the tyre to roll over the uneven surfaces. Rolling resistance is high for wet surfaces.

\subsection{Tyre Material}

Synthetic rubber is having more rolling resistance than natural rubber. Butyl rubber has more rolling resistance than synthetic rubber but has good road holding and traction properties.

\subsection{Inflation Pressure}

Depending on the road type inflation pressure affects the rolling resistance. On hard surfaces when inflation pressure increases rolling resistance decreases. On deformable road surfaces more inflation pressure results in high ground penetration and more work is to be done by the tyre. If the inflation pressure is less, more deflection occurs resulting in high rolling resistance. So an optimum tyre pressure is to be maintained.

\subsection{Speed}

As speed of the rolling tyre increases the work done in deforming the tyre also increases and hence the rolling resistance increases. The multitude of factors discussed previously lead to complexity in the determination of rolling resistance. However in performance calculations it is sufficient to express co-efficient of rolling resistance as a linear function of speed.

Co-efficient of rolling resistance $\left(f_{r}\right)$ is the ratio between the Rolling resistance force $\left(\boldsymbol{F}_{\boldsymbol{r}}\right)$ and the vertical force $\left(\boldsymbol{F}_{z}\right)$

$$
\begin{aligned}
& f_{r}=\frac{F_{r}}{F_{z}} \\
& f_{r}=0.01\left(1+\frac{v_{c o g}}{100}\right)
\end{aligned}
$$

We know,

$$
\begin{gathered}
F_{a c c}=m_{c o g} \dot{v}_{c o g} \\
m_{c o g} \dot{v}_{c o g}=F_{d r i v e}-F_{w i n d x}-F_{r}-F_{g}
\end{gathered}
$$

The accelerating force is not only used for the vehicle acceleration. It should also accelerate rotary parts of the driveline and the wheels. Hence a mass factor should be introduced to compensate for the effect. Thus equation 3.40 becomes

$$
\begin{gathered}
m_{\text {cog }} \cdot f_{\text {mass }} \cdot \dot{v}_{\text {cog }}=F_{\text {drive }}-F_{\text {windx }}-F_{r}-F_{g} \\
m_{\text {cog }}\left(f_{\text {mass }} \cdot \dot{v}_{\text {cog }}+g \cdot \chi_{\text {road }}\right)=F_{\text {drive }}-F_{\text {windx }}-F_{r} \\
m_{\text {cog }}=\frac{F_{\text {drive }}-F_{\text {windx }}-F_{r}}{f_{\text {mass }} \dot{v}_{\text {cog }}+g \chi_{\text {road }}}
\end{gathered}
$$

\section{Equations of Motion}

The eleven equations for the various movements of the vehicle are given as follows

$$
\begin{aligned}
& m \ddot{y}+2 d_{y} \dot{y}-2 d_{y} \dot{y}_{w f l}-2 d_{y}\left(h_{c}-h_{c e}\right) \dot{\phi}-2 h_{c e} d_{y} \dot{\psi}_{w f l} \\
& +2 k_{y} y-2 k_{y} y_{w f l}-2 k_{y}\left(h_{c}-h_{c e}\right)-2 h_{c e} k_{y} \psi_{w f l}=0
\end{aligned}
$$




$$
\begin{aligned}
& J_{x} \ddot{\phi}+2\left(h_{c}-h_{c e}\right) d_{y} \dot{y}_{w f l}+2\left[\left(h_{c}-h_{c e}\right)^{2} d_{y}+h_{c e}{ }^{2} d_{y}+l_{c}^{2} d_{z}\right] \\
& \dot{\phi}-2 l_{c}^{2} d_{y} \dot{\psi}_{w f l}+2\left(h_{c}-h_{c e}\right) k_{y} y_{w f l}+2\left(h_{c}-h_{c e}\right)^{2} k_{y} \phi+2 h_{c e}^{2} k_{y} \psi_{w f l} \\
& m_{w r r} \ddot{y}_{w r r}-\left(d_{w y}+\frac{b_{f r}^{2}}{b_{r}^{2}}\right) \dot{y}_{w r r}-\frac{\left(m_{c o g}-m_{u n}\right) i_{x}^{2} V}{r_{s t a t} b_{r}} \dot{\psi}_{w r r} \\
& +2 l_{c}^{2} k_{z} \phi-2 l_{c}^{2} k_{z} \psi_{w f l}=0 \\
& J_{z} \ddot{\psi}+2 l_{c}^{2} d_{y} \dot{\psi}-l_{c} d_{y} \dot{y}_{w f l}+2 l_{c}^{2} k_{y} \psi-l_{c} k_{y} y_{w f l}=0 \\
& -d_{u y} \dot{y}+\left(h_{p}^{\prime} d_{u y}+\frac{b_{f r}^{2}}{b_{r}^{2}} d_{d u}\right) \dot{\phi}-l_{r} d_{u y} \dot{\psi}+\left(k_{w y}+\frac{b_{f r}^{2}}{b_{r}^{2}} k_{w z}\right) y_{w r r} \\
& -l_{r} k_{u y} \psi-k_{u y} y+\left(h_{p}^{\prime} k_{u y}+\frac{b_{f r}^{2}}{b_{r}^{2}} k_{u z}\right) \phi+k_{t y} y_{w r r}=0 \\
& m_{w f l} \ddot{y}_{w f l}+\left(d_{w y}+\frac{b_{f s}^{2}}{b_{f}^{2}}\right) \dot{y}_{w f l}+i_{x}^{2} \frac{\left(m_{c o g}-m_{u n}\right) V}{r_{s t a t} b_{f}} \dot{\psi}_{w f l}-d_{u y} \dot{y} \\
& +\left(h_{p}^{\prime} d_{u y}+\frac{b_{f s}^{2} d_{u}}{b_{f}^{2}}\right) \dot{\phi}+l_{f} d_{u y} \dot{\psi}+\left(k_{w y}+\frac{b_{f s}^{2}}{b_{f}^{2}} k_{w z}\right) y_{w f l} \\
& J_{z r r} \ddot{\psi}_{w r r}+\frac{\left(m_{c o g}-m_{u n}\right) i_{x}^{2} V}{r_{s t a t} b_{r}} \dot{y}_{w r r}+\left(b_{f r}^{2} d_{w x}\right) \dot{\psi}_{w r r} \\
& -b_{f r}^{2} d_{u x} \dot{\psi}+b_{f r}^{2} k_{w x} \dot{\psi}_{w r r}-b_{f r} k_{u x} \psi=0
\end{aligned}
$$

$+l_{f} k_{u y} \psi-k_{u y} \dot{y}+\left(h_{p}^{\prime} k_{u y}+\frac{b_{f s}^{2}}{b_{f}^{2}} k_{u z}\right) \phi+k_{t y} y_{w f l}=0$

1.11 This can be expressed in matrix form as:

$$
\begin{aligned}
& J_{z w f l} \ddot{\psi}_{w f l}+\frac{\left(m_{c o g}-m_{u n}\right) i_{x}^{2} V}{r_{s t a t} b_{f}} \dot{y}_{w f l}+\left(b_{f s}^{2} d_{w x}\right) \dot{\psi}_{w f l} b_{f s}^{2} d_{u x} \dot{\psi} \\
& +b_{f s}^{2} k_{w x} \psi_{w f l}-b_{f s}^{2} k_{u x} \psi=0
\end{aligned}
$$

$m_{w f r} \ddot{y}_{w f r}-\left(d_{w y}+\frac{b_{f s}^{2}}{b_{f}^{2}}\right) \dot{y}_{w f r}-\frac{\left(m_{c o g}-m_{u n}\right) i_{x}^{2} V}{r_{s t a t} \cdot b_{f}} \dot{\psi}_{w f r}-d_{u y} \dot{y}$ $+\left(h_{p}^{\prime} d_{u y}+\frac{b_{f s}^{2}}{b_{f}^{2}} d_{u}\right) \dot{\psi}-l_{f} d_{u y} \dot{\psi}+\left(k_{w y}+\frac{b_{f s}^{2}}{b_{f}^{2}} k_{w z}\right) y_{w f r}$

$-l_{f} k_{u y} \psi-k_{u y} y+\left(h_{p}^{\prime} k_{u y}+\frac{b_{f s}^{2}}{b_{f}^{2}}\right) \phi+k_{t y} y_{w f r}=0$

$$
\begin{gathered}
J_{z w r r} \ddot{\psi}_{w f r}+\frac{\left(m_{c o g}-m_{u n}\right) i_{x}^{2} V}{r_{s t a t} b_{f}} \dot{y}_{w f r}+\left(b_{f s}^{2} d_{w x}\right) \dot{\psi}_{w f r} \\
-b_{f s}^{2} d_{u x} \dot{\psi}+b_{f s}^{2} k_{w x} \psi_{w f r}-b_{f s}^{2} k_{u x} \psi=0
\end{gathered}
$$

$$
\begin{aligned}
& m_{w r l} \ddot{y}_{w r l}+\left(d_{w y}+\frac{b_{f r}^{2}}{b_{r}^{2}}\right) \dot{y}_{w r l}+\frac{\left(m_{c o g}-m_{u n}\right) \dot{i}_{x}^{2} V}{r_{s t a t} b_{r}} \dot{\psi}_{w r l} \\
& -d_{u y} \dot{y}+\left(h_{p}^{\prime} d_{u y}+\frac{b_{f r}^{2}}{b_{r}^{2}} d_{u}\right) \dot{\phi}+l_{r} d_{u y} \dot{\psi}+\left(k_{w y}+\frac{b_{f r}^{2}}{b_{r}^{2}} k_{w z}\right) y_{w r l} \\
& -l_{r} k_{u y} \psi-k_{u y} y+\left(h_{p}^{\prime} k_{u y}+\frac{b_{f r}^{2}}{b_{r}^{2}} k_{u z}\right) \phi+k_{t y} y_{w r l}=0
\end{aligned}
$$

$$
\begin{gathered}
J_{z w r l} \ddot{\psi}_{w r l}+\frac{\left(m_{c o g-} m_{u n}\right) i_{x}^{2} V}{r_{s t a t} b_{r}} \dot{y}_{w r l}+\left(b_{f r}^{2} d_{w x}\right) \dot{\psi}_{w r l} \\
-b_{f r}^{2} d_{u x} \dot{\psi}+b_{r}^{2} k_{w x} \psi_{w r l}-b_{r}^{2} k_{u x} \psi=0
\end{gathered}
$$$$
\left[\begin{array}{ccccccccccc}
m_{1,1} & 0 & 0 & 0 & 0 & 0 & 0 & 0 & 0 & 0 & 0 \\
0 & m_{2,2} & 0 & 0 & 0 & 0 & 0 & 0 & 0 & 0 & 0 \\
0 & 0 & m_{3,3} & 0 & 0 & 0 & 0 & 0 & 0 & 0 & 0 \\
0 & 0 & 0 & m_{4,4} & 0 & 0 & 0 & 0 & 0 & 0 & 0 \\
0 & 0 & 0 & 0 & m_{5,5} & 0 & 0 & 0 & 0 & 0 & 0 \\
0 & 0 & 0 & 0 & 0 & m_{6,6} & 0 & 0 & 0 & 0 & 0 \\
0 & 0 & 0 & 0 & 0 & 0 & m_{7,7} & 0 & 0 & 0 & 0 \\
0 & 0 & 0 & 0 & 0 & 0 & 0 & m_{8,8} & 0 & 0 & 0 \\
0 & 0 & 0 & 0 & 0 & 0 & 0 & 0 & m_{9,9} & 0 & 0 \\
0 & 0 & 0 & 0 & 0 & 0 & 0 & 0 & 0 & m_{10,10} & 0 \\
0 & 0 & 0 & 0 & 0 & 0 & 0 & 0 & 0 & 0 & m_{11,11}
\end{array}\right]\left[\begin{array}{c}
\ddot{y} \\
\ddot{\phi} \\
\ddot{\psi} \\
\ddot{y}_{w f l} \\
\ddot{\psi}_{w f l} \\
\ddot{y}_{w r r} \\
\ddot{\psi}_{w r r} \\
\ddot{y}_{w r l} \\
\ddot{\psi}_{w r l l} \\
\ddot{\psi}_{w r r}
\end{array}\right]+
$$$$
\left[\begin{array}{ccccccccccc}
c_{1,1} & c_{1,2} & 0 & c_{1,4} & c_{1,5} & 0 & 0 & 0 & 0 & 0 & 0 \\
0 & c_{2,2} & 0 & c_{2,4} & c_{2,5} & 0 & 0 & 0 & 0 & 0 & 0 \\
0 & 0 & c_{3,3} & c_{3,4} & 0 & 0 & 0 & 0 & 0 & 0 & 0 \\
c_{4,1} & c_{4,2} & c_{4,3} & c_{4,4} & c_{4,5} & 0 & 0 & 0 & 0 & 0 & 0 \\
0 & 0 & 0 & c_{5,4} & c_{5,5} & 0 & 0 & 0 & 0 & 0 & 0 \\
c_{6,1} & 0 & c_{6,3} & 0 & 0 & c_{6,6} & c_{6,7} & 0 & 0 & 0 & 0 \\
0 & 0 & c_{7,3} & 0 & 0 & c_{7,6} & c_{7,7} & 0 & 0 & 0 & 0 \\
0 & c_{8,2} & c_{8,3} & 0 & 0 & 0 & 0 & c_{8,8} & c_{8,9} & 0 & 0 \\
0 & 0 & c_{9,3} & 0 & 0 & 0 & 0 & c_{9,8} & c_{9,9} & 0 & 0 \\
0 & c_{10,2} & c_{10,3} & 0 & 0 & 0 & 0 & 0 & 0 & c_{10,10} & c_{10,11} \\
0 & 0 & c_{11,3} & 0 & 0 & 0 & 0 & 0 & 0 & c_{11,10} & c_{11,11}
\end{array}\right]\left[\begin{array}{c}
\dot{y} \\
\dot{\phi} \\
\dot{\psi} \\
\dot{y}_{w f l} \\
\dot{\psi}_{w f l} \\
\dot{y}_{w f r} \\
\dot{\psi}_{w f r} \\
\dot{y}_{w r l} \\
\dot{\psi}_{w r l} \\
\dot{y}_{w r r} \\
\dot{\psi}_{w r r}
\end{array}\right]+
$$ 


\section{Conclusion}

With the help of the above program the critical speed variations with respect to (a) Wheel base (b) Unstrung mass (c) Longitudinal damping (d) Vertical Damping (e) Longitudinal stiffness (f) Lateral Stiffness (g) Vertical stiffness (h) Position of COG and (i) Wheel track and (j) Wheel radius for the LCV(cargo) have been analyzed. It is found that the following parameters such as (a) Wheel base (b) Unstrung mass (c) Vertical damping (d) Lateral stiffness (e) Wheel track ( $\mathrm{f}$ ) Position of Centre of gravity and (g) Wheel radius have more influence on critical speed variations, are easily analyzed with computer modeling.

\section{References}

1. Allen R W and Azostak H T (1991). Characteristics influencing ground vehicle lateral/directional dynamic stability, SAE paper 910234, Society of Automotive Engineers, Warrendale, PA.

2. Cannand B E, Hathway R B et al. (1995). Critical suspension relationship and their influence on transient behavior of vehicles, Society of Automotive Engineers, 1880-1898.

3. Besinger F H, Cebon D et al. (1995). Damper models for heavy vehicle ride dynamics, Vehicle System Dynamics, vol 1(1), 35-64.

4. Blundell M V (2000). The modelling and simulation of vehicle handling part 4: handling simulation, Journal of Multi-body Dynamics, Proceedings of the Institution of Mechanical Engineers, Part K, vol 214 (2), 1-32.

5. Choromanski W (1988). Simulation researches of mathematical models of non conventional railway bogies, Engineering Archives of Transport Quarterly, New Delhi, India, 435-441.

6. Hallum C (2002). Dynamic traction characteristics of tires, SAE International, 295-2302.

7. Clark S K (1981). Mechanics of pneumatic tires, Washington D.C.

8. Clover C L and Bernard J E (1993). The influence of lateral load transfer distribution on directional response, SAE Paper 930763.

9. Dhar S D, Hohnstadt W E et al. (2002). Integrated modular methodology_-philosophy and strategy to build full vehicle finite element model, GM Technical Report, 1-67.

10. Dixon J C (1996). Tyres suspension and handling, SAE Inc., Warrendale.

11. Ono E, Asano K et al. (2003). Estimation of automotive tire force characteristics using wheel velocity, Control Engineering Practice, 1361-1370.

12. Lowndes E M (1998). Development of an intermediate dof vehicle dynamics model for optimal design studies, Ph.D Dissertation submitted to the Faculty of the North Carolina State University,
13. Fukushima N, Hidaka K et al. (1983). Optimum characteristics of automotive shock absorbers under various driving conditions and road surfaces, International Journal of Vehicle Design, vol 4(5), 463-472.

14. Heydinger G J, Bixel R A et al. (1998). Effects of loading on vehicle handling, Society of Automotive Engineers, Inc., 407-415.

15. Gim G and Nikravesh P E (1990). An analytical model of pneumatic tyres for vehicle dynamic simulations, Part 1 : pure slips, International Journal of Vehicle Design, vol 11(6), 589-618.

16. Godthelp H (1984). Studies on human vehicle control, dissertation, institute for perception TNO, NL-Soesterberg.

17. Gustafsson F (1998). Monitoring tire-road friction using tire wheel slip, IEEE Control Systems, vol 18(4), 42-49.

18. Gim G and Nikravesh P E (1990). An analytical model of pneumatic tyres for vehicle dynamics simulations, International Journal of Vehicle Design, vol11(6), 589-618.

19. Hegazy S, Rahnejat H et al. (2000). Multi-body dynamics in full vehicle handling analysis under transient maneuver, Vehicle System Dynamics, vol 34(1), 1-24.

20. Iacovoni D H (1969). Fundamentals of automobile handling analysis, Warrendale, PA: Society of Automotive Engineers, Inc.

21. Svendenius J and Wittenmark B (2003). Brush tire model with increased flexibility, European Control Conference.

22. Kiencke U (1993). Realtime estimation of adhesion characteristic between tyres and road, $12^{\text {th }}$ IFAC World Congress of Automatic Control, vol 1, 15-11, Sydney.

23. Kiencke U, and Nielsen L (2000). Automotive Control Systems for Engine, Chapter 10.1088, Driveline and Vehicle, Springer.

24. Suh K, Lee Y et al. (2002). A study on the handling performances of a large-sized bus with the change of rear suspension geometry, SAE International, 648-667.

25. Lee S W (1994). Development of new dynamic tire model for improved vehicle dynamics simulation, Ph.D. Dissertation, The Ohio State University.

26. Lozia Z (1998). Vehicle dynamics and motion simulation versus experiment, SAE Paper no. 980220, SAE Transactions, Passenger Cars, Section 6, vol 107, 344-360.

27. Dohi M and Maruyama Y (1990). Ride comfort optimization for commercial trucks, Isuzu Motors Ltd., 890-908.

28. Negrut D, Freeman J S (1994). Dynamic tire modeling for application with vehicle simulations incorporating terrain, SAE Paper 940223.

29. Flores-Centeno O, Fabela-Gallegos M J et al. (2004). Effect of wheelbase variation on the dynamic behavior of a three axles, heavy duty truck, SAE International, 120-124.

30. Pacejka H B (1993). The magic formula tyre model, Vehicle System Dynamics, vol 21(Supplement 1), 1-18.

31. Peng- Xi'an $X$, and Xie-Xi'an $Y, A$ tire traction modeling for use in ice mobile, SAE Journal Number: 1999-01-0478. 
32. Renner T E and Barber A J (2000). Accurate tire models for vehicle handling uses the empirical dynamics method, MTS systems Corp., 2000 International ADAMS Users Conference.

33. Sakai H (1981). Theoretical and experimental studies on the dynamic properties of tyres, International Journal of Vehicle Design., vol 3(3), 333-375.

34. Sakai H (1994). Measuring and Visualization of Contact Pressure Distribution of Rubber and Tyre, 13th Annual Conference, Tire Science and Technology, vol 23(4), 238-255.

35. Clark S K (1971). Mechanics of pneumatic tires, Department of Transportation, U.S. Government Printing Office, 122, 844.

36. Kimbrough S S (1999). Rule-based wheel slip assignment for vehicle stability enhancement, SAE Journal No: 1999-01-0476.

37. Duym S W R (2000). Simulation tools, modeling and identification, for an automotive shock absorber in the context of vehicle dynamics, Vehicle System Dynamics, vol 33(4), 261-285.
38. Verros G, Georgiou G et al. (2005). Multi-objective optimization of quarter-car models with linear or piecewise linear suspension dampers, ASME International Design Engineering Technical Conferences and Computers and Information in Engineering Conference, DETC2005-85232.

39. Winkler C B et al. (1992). Roll-stability performance of heavy-truck suspensions, SAE Paper No. 922426.

40. Kang X and Deng W (2007). Vehicle-trailer handling dynamics and stability control - an engineering review, SAE 2007-01-0822.

41. Zhang Y, Palmer T J et al. (1998). Vehicle chassis/suspension dynamics analysis-finite element model versus rigid body mode, SAE Journal No - 980900.

42. Zardecki D (1998). Mathematical model of car steering system dynamics with regard to gear backlash and friction, Proceedings of VI International Conference Autoprogres'98, $43-52$. 